\title{
Intrathecal and Intraventricular Administration of Antibiotics in Gram-Negative Nosocomial Meningitis in a Research Hospital in Turkey
}

\author{
Muge AYHAN ${ }^{1}$, Ayse KAYA KALEM ${ }^{2}$, Imran HASANOGLU², Bircan KAYAASLAN ${ }^{2}$, Mehmet Ozgur OZATES ${ }^{3}$, \\ Seval IZDES ${ }^{4}$, Burcin HALACLI ${ }^{5}$, Hatice Rahmet GUNER ${ }^{2}$
}

\begin{abstract}
${ }^{1}$ Ankara City Hospital, Department of Infectious Diseases and Clinical Microbiology, Ankara, Turkey
${ }^{2}$ Ankara Yildirim Beyazit University, Department of Infectious Diseases and Clinical Microbiology, Ankara, Turkey

${ }^{3}$ Ankara City Hospital, Department of Neurosurgery, Ankara, Turkey

${ }^{4}$ Ankara Yildirim Beyazit University, Department of Anaesthesia and Intensive Care, Ankara, Turkey

${ }^{5}$ Hacettepe University, School of Medicine, Department of Internal Medicine, Division of Intensive Care, Ankara, Turkey

This study has been presented as an oral presentation at the BUHASDER Congress between 16 and 20 October 2019 at Izmir, Turkey.
\end{abstract}

Corresponding author: Muge AYHAN dr.mugeayhan@hotmail.com

\section{ABSTRACT}

AIM: To evaluate the gram-negative nosocomial meningitis cases which were treated with intrathecal (IT) / intraventricular (IVT) antibiotics.

MATERIAL and METHODS: Medical records were reviewed for IT/IVT antibiotherapy. Gram-negative nosocomial meningitis cases treated with IT/IVT antibiotherapy additional to systemic antibiotics were included. All patients' sex, age, SOFA scores, surgical history, cerebrospinal fluid (CSF) culture results, CSF cell counts, systemic and IT/IVT antibiotics, their dosages and duration, CSF culture sterility and sterility time, 28-day mortality due to meningitis, and all other causes were recorded and analyzed.

RESULTS: Thirteen patients were included between 2014 and 2018. Most common microorganism was Acinetobacter baumannii (A.baumannii) (8/13). IT/IVT antibiotics were chosen according to susceptibility. Colistin was used in eight patients, amikacin was used in four, and one patient used amikacin and colistin consecutively. Culture negativity could not be achieved in two patients. Eight patients clinically improved but five patients had no clinical response. 28-day mortality due to infection occured in 2 of 13 patients (15\%). 28-day all-cause mortality occured in 3 of 13 patients (23\%).

CONCLUSION: In our study, CSF culture negativity rate was high. IT/IVT antibiotic therapy should be considered as an effective and acceptable treatment option, especially in patients who do not respond to standard IV antibiotherapy.

KEYWORDS: Meningitis, Ventriculitis, Intrathecal, Intraventricular, Antibiotics

ABBREVIATIONS: IV: Intravenous, IT: Intrathecal, IVT: Intraventricular, SOFA: Sequential organ failure assessment, CSF: Cerebrospinal fluid, CNS: Central nervous system

Muge AYHAN $\quad$ (D) : 0000-0002-4821-5559

Ayse KAYA KALEM (D) : 0000-0002-4759-0066

Imran HASANOGLU (D) : 0000-0001-6692-3893
Bircan KAYAASLAN

Mehmet Ozgur OZATES

Seval IZDES
(D) : 0000-0001-5225-8319

(D) : $0000-0002-2051-7766$

(D) : 0000-0001-9856-2391
Burcin HALACLI

(D) : 0000-0002-7216-7438

Hatice Rahmet GUNER (D) : 0000-0002-1029-1185 


\section{- INTRODUCTION}

$\mathrm{B}$ lood-brain barrier protects central nervous system (CNS) against microbial entry from blood, while skull and leptomeninges protect it externally against entry of microorganisms. But in some situations, pathogens may enter CNS through a breakdown of blood-brain barrier or by direct invasion through external barriers (23). Nosocomial CNS infections can be complications of neurosurgical procedures or they can occur spontaneously. Nosocomial bacterial meningitis can be caused by internal or external drainage catheter placement, lumbar puncture, intrathecal infusion of medications, or spinal anesthesia and complicated head trauma (12). They can lead to important neurological complications and cause increased mortality. They can also result in longer hospital stay and increased costs $(5,14)$. Reported rates of CNS infections depend on intervention type (4). Nosocomial meningitis occurs in 0.8 to $1.5 \%$ of patients who undergo cranial surgery. Post-operative meningitis represents nearly $30 \%$ of all post-neurosurgery infections. Carbapenem resistant gram-negative post-operative meningitis is associated with high mortality rates up to $60-70 \%$ (13). Clinically it can be difficult to diagnose. Normal CSF cell count, protein and/ or glucose, and a negative CSF gram-stain do not exclude the presence of infection. CSF cultures are the most important tests to establish the diagnosis (7). The causative agent is frequently coagulase negative staphyloccocci amongst gram-positives. Amongst gram-negative rods, A.baumannii, aerobic gram negative enteric microorganisms (e.g Klebsiella pneumoniae (K.pneumoniae)) can be seen. A gram-negative origin is associated with severe underlying conditions and poor outcome (18). Risk factors for meningitis or ventriculitis are leakage of CSF, concominant infection at the time of cranial surgery, prolonged or repeated surgery, prolonged ventricular drainage, surgery through the sinuses, and the severity of underlying diseases (1). These infections are complex and difficult to treat because bacteria are becoming increasingly resistant to the most commonly used antibiotics. Another challenge is that attaining effective antibiotic concentration within the CNS is difficult, because blood-brain barrier decreases antibiotic penetration from reaching the desired area. Various approaches have been tried to achieve therapeutic levels in the central nervous system. Some of these approaches are, increasing antibiotic dosage, choosing small molecular weight antibiotics, and using antibiotics which bind to plasma proteins less. Despite all these strategies, it is difficult to reach the appropriate drug concentration within the CNS without systemic toxicity (14). This has led to increased use of intrathecal (IT) or intraventricular (IVT) antibiotics to treat CNS infections effectively. Similarly, IT antibiotics have been studied in severe meningitis (with or without ventriculitis) $(8,9,19,20)$. In a randomized controlled study, which studied the effectiveness and safety of IT antibiotherapy and performed on infants who has gram-negative meningitis, the use of IT antibiotics in addition to intravenous (IV) antibiotics resulted in a 3-fold increase in relative mortality compared to standard antibiotic regimen (18). Therefore, IT antibiotic therapy should only be used as a last option if standard IV treatment fails or is likely to fail due to in vitro susceptibility testing (15). In this study, we aimed to evaluate cases of patients who received IT antibiotherapy for gram-negative nosocomial meningitis in our hospital.

\section{MATERIAL and METHODS}

This study was performed in Ankara Atatürk Research and Training Hospital. All patients, who were consulted to infectious diseases and clinical microbiology department, were retrospectively screened through consultation forms for nosocomial meningitis with previous IT or IVT antibiotic therapy. Thirteen cases were found between 2014 and 2018. All patients' sex, age, SOFA scores, surgical history, CSF culture results, CSF cell counts, systemic and IT/IVT antibiotics, their dosages and duration, CSF culture sterility, CSF culture sterility time, 28-day mortality due to meningitis, and all other causes were recorded in a follow-up questionnaire. All patients were treated empirically with IV antibiotics. Antibiotic treatment was later adjusted according to culture results. The CSF analysis was repeated once a week until CSF culture was negative. If the control CSF culture was positive, IT or IVT antibiotics were added. After 48-72 hours control of CSF culture, the process was repeated in 2-day intervals until sterility of CSF culture was achieved.

This study was approved by Ethics Committee of Clinical Research of Yıldırım Beyazıt University (Approval Date: 29 May 2019, Approval Number: 73). The study was carried out according to principles of Helsinki Declaration. Informed consent was not obtained because this study was designed as a retrospective study.

\section{Antibiotic Administration}

Patients' CSF culture results and antibiotic susceptibility results were evaluated. Systemic and IT/IVT antibiotic therapy were given together. All IT/IVT antibiotics were given at 24hour intervals. Two antimicrobial agents were used for IT/IVT treatment. Colistin was given at a dose of $10 \mathrm{mg} /$ day, while amikacin was given at a dose of $30 \mathrm{mg} /$ day. If patient has extraventricular drainage catheter (EVD), antibiotic was given through the catheter. Otherwise, it was given with daily lumbar punctures.

\section{RESULTS}

Between 2014 and 2018, 13 patients were given IT/IVT antibiotherapy. Eleven patients were male (84\%). The median age was 54 (16-65). Seven patients were followed in neurosurgical intensive care unit (ICU), four were followed in anesthesiology and reanimation ICU, and two were followed in medical ICU. All patients were intensive care patients and mean SOFA score was 5.7. Eleven patients had had cranial surgery (84\%). Six of 13 patients had EVD. Eight patients had A.baumannii growth on CSF, and 5 had K.pneumoniae (Table I). Table II shows the antimicrobial resistance of causative microorganisms. All patients had developed clinical and laboratory signs of central nervous system infection. Systemic IV antibiotics were administered in all patients after CSF culture results were obtained. Majority of patients (12/13) was given combined antibiotic therapy with different combinations. The most common IV antibiotics given to patients were 


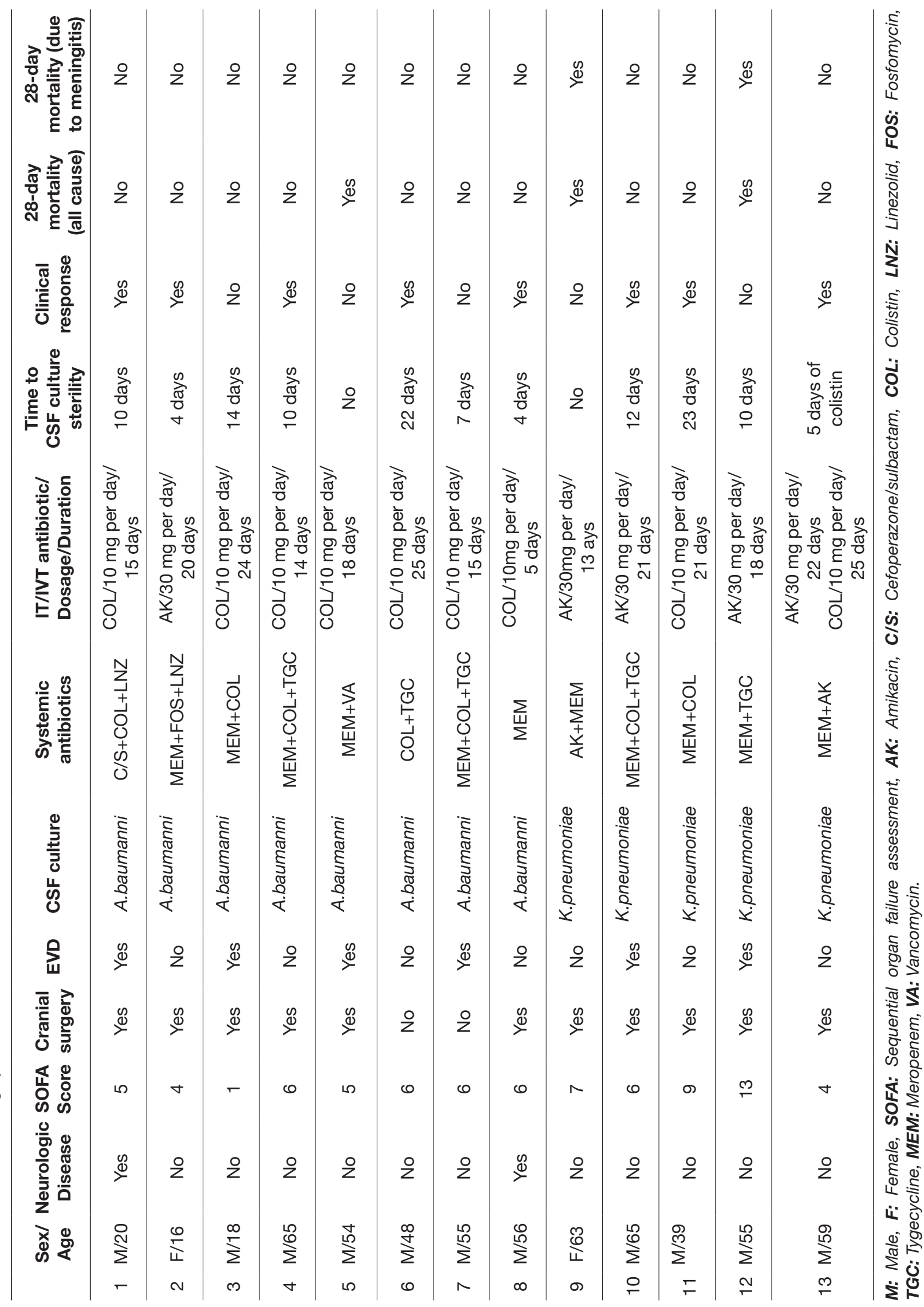


Ayhan M. et al: Intrathecal and Intraventricular Antibiotics

Table II: Antimicrobial Resistance of Causative Microrganisms Isolated from CSF Cultures

\begin{tabular}{lccc}
\hline Antimicrobial agent & A.baumanni $(\mathbf{n}=\mathbf{8})$ & K.pneumoniae $(\mathbf{n}=\mathbf{5})$ & Total $\mathbf{n = 1 3}(\mathbf{\%})$ \\
\hline Amikacin & 1 & 0 & $1(7)$ \\
\hline Carbapenems & 6 & 4 & $10(76)$ \\
\hline Cephalosporins & 8 & 4 & $12(92)$ \\
\hline Cotrimoxazole & 8 & 5 & $13(100)$ \\
\hline Gentamycin & 2 & 3 & $5(38)$ \\
\hline Colistin & 1 & 2 & $3(23)$ \\
\hline Quinolones & 8 & 5 & $13(100)$ \\
\hline
\end{tabular}

meropenem (11/13) and colistin (7/13). IT/IVT antibiotics were added to therapy, if the culture positivity persisted despite IV antibiotic treatment. IT/IVT antibiotics were chosen according to susceptibility of the microorganism. Patients who had A. baumannii meningitis received IT/IVT colistin (7/8), except for one patient who had IT/IVT amikacin due to antibiotic susceptibility results. Patients who had K.pneumoniae meningitis received IT/IVT amikacin and colistin. Three of them received amikacin, one received colistin, and one received amikacin and colistin consecutively. Mean duration of IT/IVT antibiotherapy was 20.14 days. Culture negativity couldn't be achieved in two patients. Mean time to CSF culture negativity was 13 days with a range of 4 to 27 days. Eight patients clinically improved, but five had no clinical response. 28-day mortality due to infection occurred in 2 of the 13 patients (15\%). 28-day all-cause mortality occurred in 3 of the 13 patients (23\%).

\section{DISCUSSION}

Nosocomial CNS infection is a life-threatening condition. These types of infections can occur after neurosurgery, lumbar puncture, spinal anesthesia, severe head trauma or after ventricular drainage catheter insertion (3). The most common pathogens are Staphylococci, however, gramnegative bacteria are responsible for $15 \%$ of CNS infections. Gram-negative bacteria have increased as nosocomial meningitis occurs and it should be considered in the choice of empirical antibiotherapy. The frequency of neurosurgical procedures is increasing and the wide use of broad spectrum antibiotics may have changed the species distribution and antibiotic resistance profiles $(12,21)$. A.baumannii is the most common gram-negative microorganism in the postneurosurgery setting, followed by K.pneumoniae (10). The attributable mortality of these infections is high with a range of $15-70 \%$ without appropriate treatment. The emergence of multi-drug resistant (MDR) and extensively drug resistant (XDR) strains have made treatment of these types of infections very difficult (21). In patients who have another CNS disease (trauma, tumor, hemorrhage, neurosurgery) which cause loss of blood brain barrier integrity, CNS drug penetration may have clinical importance (18). When intravenous antibiotic treatment fails, IT or IVT antibiotic administration is needed.
Therefore, IT or IVT administration of antibiotics has become an increasingly common method for the treatment of MDR or XDR-microorganism-associated CNS infections (4). Current IDSA practical guidelines for healthcareassociated ventriculitis and meningitis suggest that IVT or IT administration of antimicrobial therapy can be considered as an option for patients with healthcare-associated ventriculitis and meningitis in which the response to the standard systemic antimicrobials is poor. Several reports showed that use of some antimicrobials (eg. Polymyxin B, colistimethate sodium, gentamicin and vancomycin) is not associated with severe or irreversible toxicity $(22,24)$. There are comparative prospective studies that reported that intraventricularly administered antimicrobials show better pharmacodynamic features and similar efficacy and safety when compared to IV antimicrobials (17).

We performed a retrospective observational study aiming to assess the efficacy and treatment results of IT/IVT antibiotic administration in our patient population. We retrospectively searched consultation forms for patients who were administered IT or IVT antimicrobial therapy. Thirteen cases were found. Identified pathogens were A.baumannii $(61.5 \%)$ and K.pneumoniae (38.4\%). Clinical response was observed in nine of patients (69\%). Several case reports and case series were reported (Table III). In previous studies, the cure rate for patients who received a combination of IV/IT antimicrobials ranged from $71.4 \%$ to $100 \%$. We had a lower response rate. This difference might be because our strains were more resistant to antibiotics and because of the severity of the underlying conditions. In four of the five patients without a clinical response, there was EVD. Three of these patients had XDR A.baumannii and two had carbapenem-resistant K.pneumoniae. Therefore, clinical response could not be obtained with IT/IVT antibiotherapy.

Wang et al. evaluated intraventricular antimicrobial therapy in postneurosurgical gram-negative meningitis. Fourteen patients were treated using a sequential combination of IV/IT antibiotherapy (25). Gentamicin, amikacin, and colistin were used intrathecally. Average duration of IT therapy was 13.3 days and mean time to CSF sterility was $6.6 \pm 4.6$ days. In our study, average duration of therapy was 20.14 days and mean time to CSF sterility was 13 days. No adverse effects 
Table III: Previous Studies of Gram-Negative Meningitis in Adults Treated with Intrathecal or Intraventricular Antibiotics

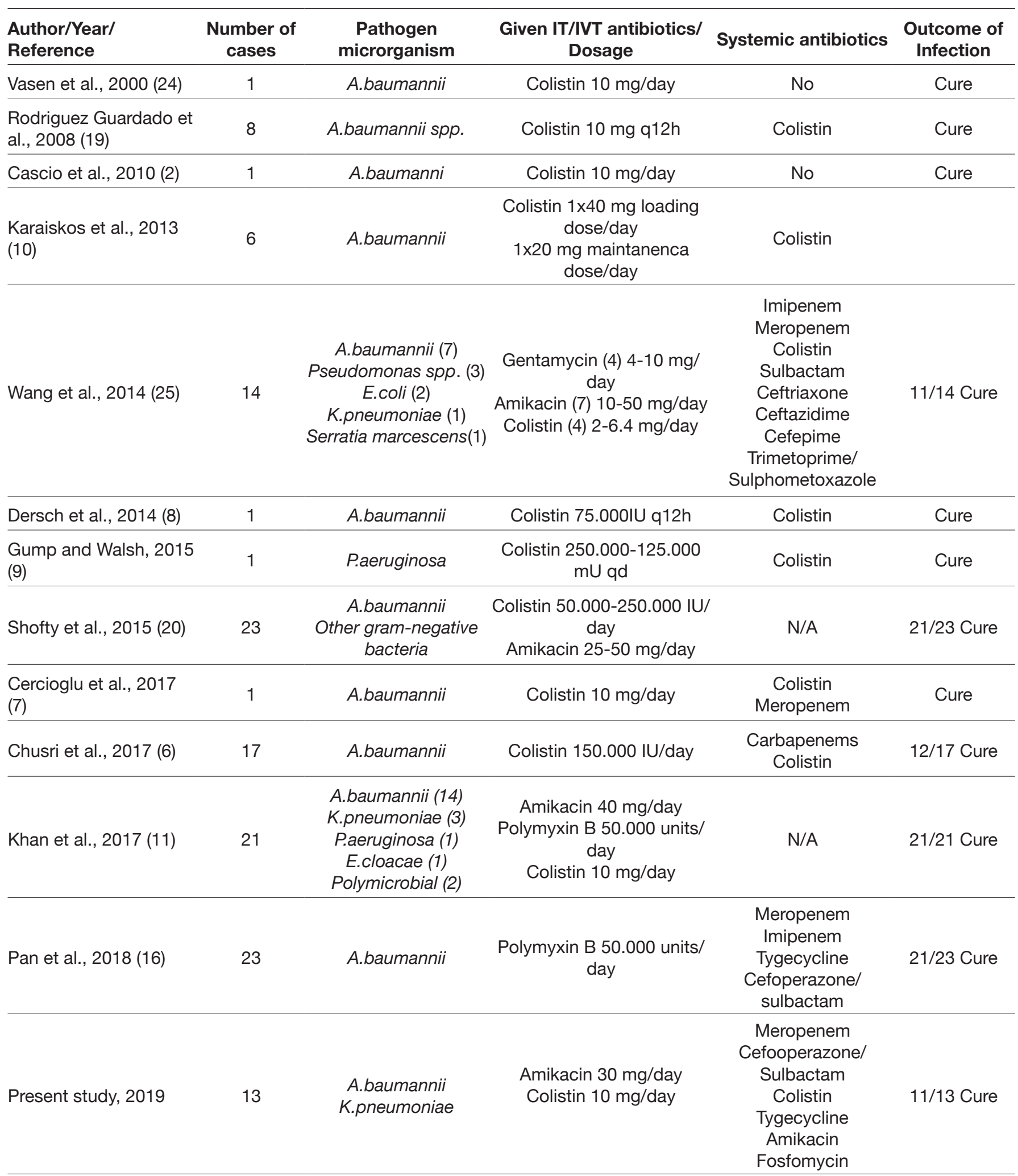

N/A: Not applicable. 
were recorded in this study during hospitalization period. The duration was longer in our study that might be due to longer period of CSF sterility. In Wang et al.'s study, no toxicity was observed in patients.

The independent risk factors for gram-negative meningitis after neurosurgery were evaluated in several studies. In a study in China, a total of 65 meningitis cases were evaluated. Diabetes, EVD, and the use of lumbar drainage were found to be independent risk factors (1). In another study, older age, emergency procedures, leak of CSF, presence of EVD, intensive care admission, repeated operations, and longer operations were independent risk factors for craniotomy meningitis (6). In our study, majority of patients (11/13, 84.6\%) have had cranial surgery, all of whom were intensive care patients and six had EVD. Our findings were consistent with the previous literature.

In a case series of 40 patients with A.baumannii meningitis, the mortality was $39 \%$, while $55 \%$ of them had carbapenem resistant isolates. Use of either intrathecal or intraventricular colistin in those with carbapenem resistance were associated with a cure of infection (13).

In the past, the major concern related to IT/IVT antimicrobial therapy was the possible adverse effects such as seizure, chemical meningitis or ventriculitis, or hearing loss $(2,6)$. No toxicity and serious adverse events were reported $(6,11)$. The first limitation of our study is the small sample size and the results were not comparable. The second with a retrospective study like our study, it is difficult to evaluate the optimal regimens, dosages, and durations for IT/IVT antibiotherapy. Further prospective randomized controlled trials are needed to evaluate the efficacy and safety of IT/IVT antibiotherapy in larger patient settings.

\section{CONCLUSION}

In conclusion, this study evaluates the clinical, microbiological outcomes of patients with A.baumannii and K.pneumoniae meningitis. In our study, CSF culture negativity rate was high. This result, with no toxicity or adverse effects, makes IT/ IVT antibiotic therapy an effective and acceptable treatment option, especially in patients who do not respond to standard IV antibiotherapy. However, further large scale randomized controlled trials are needed.

\section{口EFERENCES}

1. Bargiacchi O, De Rosa FG: Intrathecal or intraventricular colistin: A review. Infez Med 24(1):3-11, 2016

2. Cascio A, Conti A, Sinardi L, laria C, Angileri FF, Stassi G, David $T$, Versaci A, laria $M$, David A: Post-neurosurgical multidrugresistant Acinetobacter baumannii meningitis successfully treated with intrathecal colistin. A new case and a systematic review of the literature. Int J Infect Dis 14(7):572-579, 2010

3. Cercioglu D, Cesur S, Ataman-Hatipoglu C, Yildirim M, Celik H, Kinikli S, Bayar MA: Nosocomial meningitis due to multidrug -resistant Acinetobacter baumannii successfully treated with intrathecal colistin. Klimik Derg 30(3):155-157, 2017
4. Ceylan B, Arslan F, Sipahi OR, Sunbul M, Ormen B, Hakyemez IN, Turunc T, Yildiz Y, Karsen H, Karagoz G, Tekin R, Hizarci B, Turhan V, Senol S, Oztoprak N, Yilmaz M, Ozdemir K, Mermer S, Kokoglu OF, Mert A: Variables determining mortality in patients with Acinetobacter baumannii meningitis/ventriculitis treated with intrathecal colistin. Clinical Neurol Neurosurg 153:43-49, 2016

5. Chen C, Zhang B, Yu S, Sun F, Ruan Q, Zhang W, Shao L, Chen S: The incidence and risk factors of meningitis after major craniotomy in China: A retrospective cohort study. PLoS One 9(7):e101961, 2014

6. Chen $\mathrm{CH}$, Chang $\mathrm{CY}$, Lin LJ, Chen WL, Chang YJ, Wang $\mathrm{SH}$, Cheng $\mathrm{CY}$, Yen HC. Risk factors associated with postcraniotomy meningitis: A retrospective study. Medicine (Baltimore) 95(31):e4329, 2016

7. Chusri S, Sakarunchai I, Kositpantawong N, Panthuwong S, Santimaleeworagun W, Pattharachayakul S, Singkhamanan K, Doi Y: Outcomes of adjunctive therapy with intrathecal or intraventricular administration of colistin for post-neuro surgical meningitis and ventriculitis due to carbepenemresistant acinetobacter baumannii. Int $\mathrm{J}$ Antimicrob Agents 51(4):646-650, 2018

8. Dersch R, Robinson E, Beume L, Rauer S, Niesen WD: Full remission in a patient with catheter-associated ventriculitis due to Acinetobacter baumannii treated with intrathecal and intravenous colistin besides coinfections with other multidrugresistant bacteria. Neurol Sci 36(4):633-634, 2015

9. Gump WC, Walsh JW: Intrathecal colistin for tratment of highly resistant Pseudomonas ventriculitis. J Neurosurg 102:915917, 2015

10. Karaiskos I, Galani L, Baziaka F, Katsouda E, loannidis I, Andreou A, Paskalis H, Giamarellou H: Successful treatment of extensively drug-resistant Acinetobacter baumannii ventriculitis and meningitis with intraventricular colistin after application of a loading dose: A case series. Int J Antimicrob Agents 41(5):480-483, 2013

11. Khan SA, Waqas M, Siddiqui UT, Shamim MS, Nathani KR, Jooma R, Mehmood F: Intrathecal and intraventricular antibiotics for postoperative Gram-negative meningitis and ventriculitis. Surg Neurol Int 8:226, 2017

12. Kim H, Kim S, Park G, Kwon E, Kim H\& Jeong JY, Chang $\mathrm{HH}$, Lee JM, Kim NS: The causes and treatment outcomes of 91 patients with adult nosocomial meningitis. The Korean Journal of Internal Medicine 27(2):171, 2012

13. Moon C, Kwak YG, Kim BN, Kim ES, Lee CS: Implications of postneurosurgical meningitis caused by carbapenemresistant Acinetobacter baumannii. J Infect Chemother 19:916-919, 2013

14. Mrowczynski OD, Langan ST, Rizk EB: Intra-cerebrospinal fluid antibiotics to treat central nervous system infections: A review and update. Clin Neurol Neurosurg 170:140-158, 2018

15. Nau R, Seele J, Djukic M, Eiffert H: Pharmacokinetics and pharmacodynamics of antibiotics in central nervous system infections. Curr Opin Infect Dis 31(1):57-68, 2018 
16. Pan S, Huang X, Wang Y, Zhao C, Yao Z, Cui W, Zhang G: Efficacy of intravenous plus intrathecal/intracerebral ventricle injection of polymyxin B for post-neurosurgical intracranial infections due to MDR/XDR Acinetobacter baumannii: A retrospective cohort study. Antimicrob Resist Infect Control $7: 8,2018$

17. Pfausler B, Spiss H, Beer R, Kampl A, Engelhardt K, Schober $\mathrm{M}$, Schmutzhard E: Treatment of staphylococcal ventriculitis associated with external cerebrospinal fluid drains: A prospective randomized trial of intravenous compared with intraventricular vancomycin therapy. J Neurosurg 98:10401044, 2003

18. Remês F, Tomăŝ R, Jindrák V, Vaniŝ V, Setlík M: Intraventricular and lumbar intrathecal administration of antibiotics in postneurosurgical patients with meningitis and/or ventriculitis in a serious clinical state. J Neurosurg 119(6):1596-1602, 2013

19. Rodriguez Guardado A, Blanco A, Asensi V, Pérez F, Rial JC, Pintado V, Bustillo E, Lantero M, Tenza E, Alvarez M, Maradona JA, Carton JA: Multidrug-resistant Acinetobacter meningitis in neurosurgical patients with intraventricular catheters: Assessment of different treatments. J Antimicrob Chemother 61(4):908-913, 2008
20. Shofty B, Neuberger A, Naffaa ME, Binawi T, Babitch T, Rappaport ZH, Zaaroor M, Sviri G, Paul M: Intrathecal or intraventricular therapy for post-neurosurgical Gram-negative meningitis: Matched cohort study. Clin Microbiol Infect 22: 66-70, 2016

21. Tsimogianni A, Alexandropoulos $P$, Chantziara V, Vassi $A$, Micha G, Lagiou F, Chinou E, Michaloudis G, Georgiou S: Intrathecal or intraventricular administration of colistin, vancomycin and amikacin for central nervous system infections in neurosurgical patients in an intensive care unit. Int J Antimicrob Agents 49(3):389-390, 2017

22. Tunkel A, Hasbun R, Bhimraj A, Byers K, Kaplan S, Scheid W, van de Beek D, Bleck TP, Garton HJL, Zunt JR: 2017 Infectious Diseases Society of America's Clinical Practice Guidelines for Healthcare-Associated Ventriculitis and Meningitis. Clinical Infectious Diseases 64(6):34-65, 2017

23. Van de Beek D, Drake JM, Tunkel AR: Nosocomial bacterial meningitis. N Engl J Med 362(2):146-154, 2010

24. Vasen W, Desmery P, Ilutovich S, Di Martino A: Intrathecal use of colistin. J Clin Microbiol 38(9):3523, 2000

25. Wang JH, Lin PC, Chou CH, Ho CM, Lin KH, Tsai CT, Wang $\mathrm{JH}$, Chi CY, Ho MW: Intraventricular antimicrobial therapy in postneurosurgical Gram-negative bacillary meningitis or ventriculitis: A hospital-based retrospective study. J Microbiol Immunol Infect 47(3):204-210, 2014 